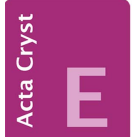
COMMUNICATIONS

ISSN 2056-9890

\section{Crystal structure of 3-chloro-N-(2-nitro- phenyl)benzamide}

\section{Rodolfo Moreno-Fuquen, ${ }^{\mathrm{a} *}$ Alexis Azcárate ${ }^{\mathrm{a}}$ and Alan R. Kennedy ${ }^{\mathbf{b}}$}

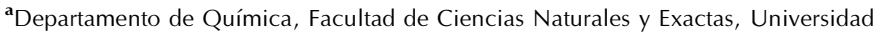
del Valle, Apartado 25360, Santiago de Cali, Colombia, and ${ }^{\mathbf{b}}$ WestCHEM, Department of Pure and Applied Chemistry, University of Strathclyde, 295 Cathedral Street, Glasgow G1 1XL, Scotland. *Correspondence e-mail: rodimo26@yahoo.es

Received 2 August 2015; accepted 3 August 2015

Edited by W. T. A. Harrison, University of Aberdeen, Scotland

In the title compound, $\mathrm{C}_{13} \mathrm{H}_{9} \mathrm{ClN}_{2} \mathrm{O}_{3}$, the mean plane of the central amide fragment (r.m.s. deviation $=0.016 \AA$ ) subtends dihedral angles of $15.2(2)$ and $8.2(2)^{\circ}$ with the chloro- and nitro-substituted benzene rings, respectively. An intramolecular $\mathrm{N}-\mathrm{H} \cdots \mathrm{O}$ hydrogen bond generates an $S(6)$ ring. In the crystal, molecules are linked by weak $\mathrm{C}-\mathrm{H} \cdots \mathrm{O}$ hydrogen bonds, forming $C(7)$ chains which propagate along [010], but no $\mathrm{Cl} \cdots \mathrm{Cl}$ short contacts are observed.

Keywords: crystal structure; benzamide; hydrogen bonding; halogenhalogen interactions.

CCDC reference: 1416793

\section{Related literature}

For halogen-halogen interactions in benzanilide compounds, see: Vener et al. (2013); Nayak et al. (2011).

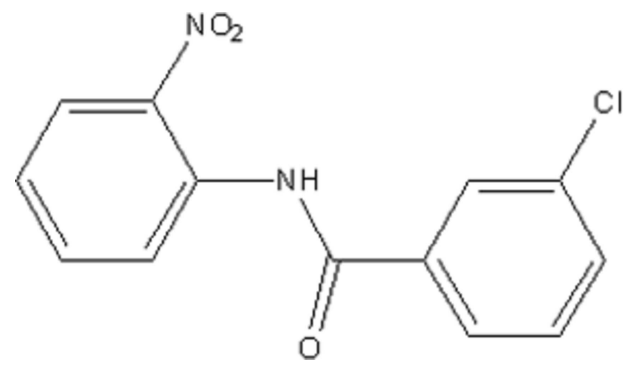

\section{Experimental}

2.1. Crystal data

$\mathrm{C}_{13} \mathrm{H}_{9} \mathrm{ClN}_{2} \mathrm{O}_{3}$
Monoclinic, $P 2_{1} / c$

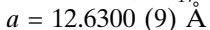

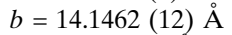

$c=6.7797(6) \AA$

$\beta=105.475(7)^{\circ}$

$V=1167.39(17) \AA^{3}$

\subsection{Data collection}

Oxford Diffraction Gemini S diffractometer

Absorption correction: multi-scan (CrysAlis PRO; Oxford
$Z=4$

Mo $K \alpha$ radiation

$\mu=0.33 \mathrm{~mm}^{-1}$

$T=123 \mathrm{~K}$

$0.40 \times 0.08 \times 0.05 \mathrm{~mm}$

\subsection{Refinement}

$R\left[F^{2}>2 \sigma\left(F^{2}\right)\right]=0.068$

$w R\left(F^{2}\right)=0.179$

$S=1.00$

10367 reflections

177 parameters
Diffraction, 2010)

$T_{\min }=0.839, T_{\max }=1.000$ 10366 measured reflections 10366 independent reflections 7015 reflections with $I>2 \sigma(I)$

Table 1

Hydrogen-bond geometry $\left(\AA{ }^{\circ}\right)$.

\begin{tabular}{lllll}
\hline$D-\mathrm{H} \cdots A$ & $D-\mathrm{H}$ & $\mathrm{H} \cdots A$ & $D \cdots A$ & $D-\mathrm{H} \cdots A$ \\
\hline $\mathrm{N} 1-\mathrm{H} 1 N \cdots \mathrm{O} 2$ & $0.98(7)$ & $1.75(7)$ & $2.612(6)$ & $144(6)$ \\
$\mathrm{C} 10-\mathrm{H} 10 \cdots \mathrm{O} 1^{\mathrm{i}}$ & 0.95 & 2.39 & $3.158(7)$ & 138 \\
\hline
\end{tabular}

Symmetry code: (i) $-x+1, y-\frac{1}{2},-z+\frac{3}{2}$.

Data collection: CrysAlis PRO (Oxford Diffraction, 2010); cell refinement: CrysAlis PRO; data reduction: CrysAlis PRO; program(s) used to solve structure: SIR92 (Altomare et al., 1994); program(s) used to refine structure: SHELXL2014 (Sheldrick, 2015); molecular graphics: ORTEP-3 for Windows (Farrugia, 2012) and Mercury (Macrae et al., 2006); software used to prepare material for publication: Win $G X$ (Farrugia, 2012).

\title{
Acknowledgements
}

RMF is grateful to the Universidad del Valle, Colombia, for partial financial support.

Supporting information for this paper is available from the IUCr electronic archives (Reference: HB7476).

\section{References}

Altomare, A., Cascarano, G., Giacovazzo, C., Guagliardi, A., Burla, M. C., Polidori, G. \& Camalli, M. (1994). J. Appl. Cryst. $27,435$.

Farrugia, L. J. (2012). J. Appl. Cryst. 45, 849-854.

Macrae, C. F., Edgington, P. R., McCabe, P., Pidcock, E., Shields, G. P., Taylor, R., Towler, M. \& van de Streek, J. (2006). J. Appl. Cryst. 39, 453-457.

Nayak, S. K., Reddy, M. K., Guru Row, T. N. \& Chopra, D. (2011). Cryst. Growth Des. 11, 1578-1596.

Oxford Diffraction (2010). CrysAlis PRO. Oxford Diffraction Ltd, Yarnton, England.

Sheldrick, G. M. (2015). Acta Cryst. C71, 3-8.

Vener, M. V., Shishkina, A. V., Rykounov, A. A. \& Tsirelson, V. G. (2013). J. Phys. Chem. A, 117, 8459-8467. 


\title{
supporting information
}

Acta Cryst. (2015). E71, o674 [https://doi.org/10.1107/S2056989015014620]

\section{Crystal structure of 3-chloro-N-(2-nitrophenyl)benzamide}

\author{
Rodolfo Moreno-Fuquen, Alexis Azcárate and Alan R. Kennedy
}

\section{S1. Comment}

The crystal structure determination of 3-chloro-N-(2-nitrophenyl)benzamide (I), is part of a study on benzanilides carried out in our research group, and it was obtained from the reaction between 3-chlorobenzoic acid and 2-nitroaniline mediated by the presence of thionyl chloride. The study of intermolecular halogen-halogen interactions is a current problem and several authors have presented interesting results. Halogen-halogen short interactions, in other similar studies, show $\mathrm{Cl} \cdots \mathrm{Cl}$ distances of the order of $3.8 \AA$. Theoretical studies of density analysis, varying the $\mathrm{Cl} \cdots \mathrm{Cl}$ distance from 3.0 to $4.0 \AA$, using DFT solid state program, have been undertaken (Vener et al., 2013). Geometric considerations in halogen-halogen interactions, for various benzanilide systems, showed different behaviors. Interactions of fluorine with other halogens $\mathrm{Cl}, \mathrm{Br}$, I, in different benzanilide systems, include interactions type: trans, cis or L-geometry (Nayak et al., 2011). The molecular structure of (I) is shown in Fig. 1. The central amide moiety, C8-N1-C7(=O1) - C1, is essentially planar (r.m.s. deviation for all non-H atoms $=0.0164 \AA$ ) and it forms dihedral angles of $15.2(2)^{\circ}$ with the $\mathrm{C} 1-\mathrm{C} 6$ and $8.2(2)^{\circ}$ with the $\mathrm{C} 8-\mathrm{C} 13$ rings respectively. In the crystal structure (Fig. 2), molecules are linked by weak $\mathrm{C}-\mathrm{H} \cdots \mathrm{O}$ intermolecular contacts. The $\mathrm{C} 10-\mathrm{H} 10 \cdots \mathrm{O} 1$ hydrogen bond interactions are responsible for crystal growth parallel to $(20$ -2). In this interaction, the $\mathrm{C}-\mathrm{H}$ in the molecule at $(\mathrm{x}, \mathrm{y}, \mathrm{z})$ acts as a hydrogen-bond donor to $\mathrm{O} 1$ atom of the carbonyl group at $(-\mathrm{x}+1,+\mathrm{y}-1 / 2,-\mathrm{z}+3 / 2)$. These interactions generate $\mathrm{C}(7)$ chains of molecules along [010]. Other intra $\mathrm{N}-\mathrm{H} \cdots \mathrm{O}$ and N$\mathrm{H} \cdots \mathrm{N}$ are observed (see Table 1, Nardelli, 1995). The shorest $\mathrm{Cl} \cdots \mathrm{Cl}$ contact distance in this structure is 3.943 (3) $\AA$.

\section{S2. Experimental}

The title molecule was synthesized taking $0.200 \mathrm{~g}(1.270 \mathrm{mmol})$ of 3-chlorobenzoic acid and it was placed under reflux with $2 \mathrm{~mL}$ of thionyl chloride for two hours. After this time an equimolar amount of o-nitroaniline, dissolved in $10 \mathrm{~mL}$ of acetonitrile and allowed to reflux at constant stirring for 3 hours was added. The final solution was left to slow evaporation to obtain yellow crystals. [m.p. 399 (1)K].

\section{S3. Refinement}

All $\mathrm{Hm}$ atoms were positioned in geometrically idealized positions, $\mathrm{C}-\mathrm{H}=0.95 \AA$, and were refined using a ridingmodel approximation with $U_{\text {iso }}(\mathrm{H})$ constrained to 1.2 times $U_{\text {eq }}$ of the respective parent atom. $\mathrm{H} 1 \mathrm{~N}$ atom was found from the Fourier maps and its coordinates were refined freely. 


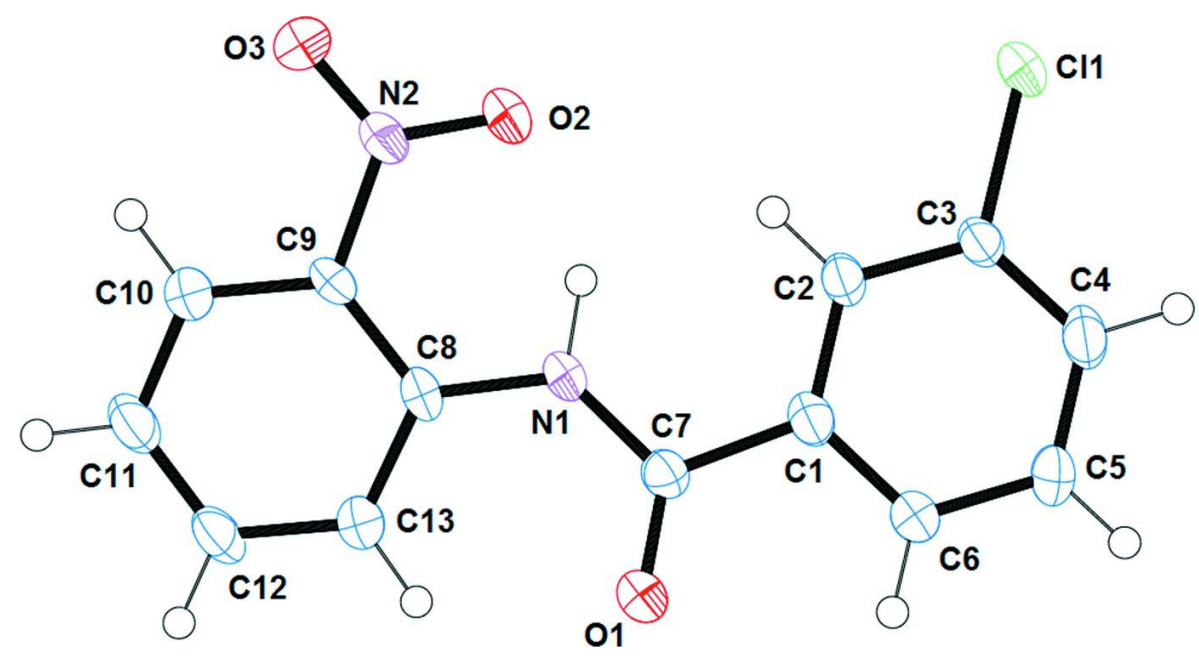

Figure 1

The molecular structure of (I) with displacement ellipsoids drawn at the $50 \%$ probability level. $\mathrm{H}$ atoms are shown as spheres of arbitrary radius.

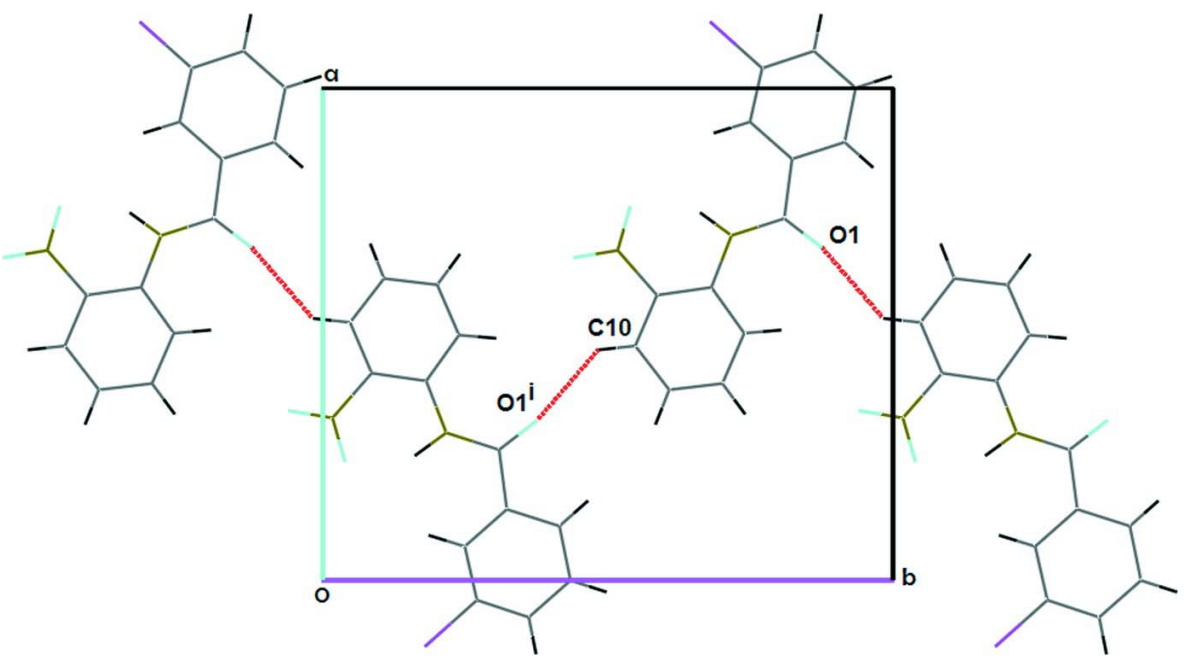

Figure 2

Part of the crystal structure of (I), showing the formation of C(7) chains along [010] [Symmetry code: (i) $-x+1, y-1 / 2,-$ $z+3 / 2]$.

\section{3-Chloro-N-(2-nitrophenyl)benzamide}

\section{Crystal data}

$\mathrm{C}_{13} \mathrm{H}_{9} \mathrm{ClN}_{2} \mathrm{O}_{3}$

$M_{r}=276.67$

Monoclinic, $P 2_{1} / c$

$a=12.6300$ (9) $\AA$

$b=14.1462(12) \AA$

$c=6.7797(6) \AA$

$\beta=105.475(7)^{\circ}$

$V=1167.39(17) \AA^{3}$

$Z=4$

$F(000)=568$
$D_{\mathrm{x}}=1.574 \mathrm{Mg} \mathrm{m}^{-3}$

Melting point: 399(1) $\mathrm{K}$

Mo $K \alpha$ radiation, $\lambda=0.71073 \AA$

Cell parameters from 10366 reflections

$\theta=3.3-27.0^{\circ}$

$\mu=0.33 \mathrm{~mm}^{-1}$

$T=123 \mathrm{~K}$

Needle, yellow

$0.40 \times 0.08 \times 0.05 \mathrm{~mm}$ 


\section{Data collection}

Oxford Diffraction Gemini S diffractometer

Radiation source: fine-focus sealed tube Graphite monochromator $\omega$ scans

Absorption correction: multi-scan (CrysAlis PRO; Oxford Diffraction, 2010) $T_{\min }=0.839, T_{\max }=1.000$

\section{Refinement}

Refinement on $F^{2}$

Least-squares matrix: full

$R\left[F^{2}>2 \sigma\left(F^{2}\right)\right]=0.068$

$w R\left(F^{2}\right)=0.179$

$S=1.00$

10367 reflections

177 parameters

0 restraints

Primary atom site location: structure-invariant direct methods
10366 measured reflections

10366 independent reflections

7015 reflections with $I>2 \sigma(I)$

$R_{\text {int }}=0.000$

$\theta_{\max }=29.0^{\circ}, \theta_{\min }=3.3^{\circ}$

$h=-17 \rightarrow 17$

$k=-17 \rightarrow 17$

$l=-9 \rightarrow 8$

Secondary atom site location: difference Fourier map

Hydrogen site location: inferred from neighbouring sites

$\mathrm{H}$ atoms treated by a mixture of independent and constrained refinement

$w=1 /\left[\sigma^{2}\left(F_{\mathrm{o}}^{2}\right)+(0.0657 P)^{2}\right]$ where $P=\left(F_{\mathrm{o}}^{2}+2 F_{\mathrm{c}}^{2}\right) / 3$

$(\Delta / \sigma)_{\max }<0.001$

$\Delta \rho_{\max }=0.78 \mathrm{e} \AA^{-3}$

$\Delta \rho_{\min }=-0.49$ e $\AA^{-3}$

\section{Special details}

Experimental. IR spectra was recorded on a FT - IR SHIMADZU IR-Affinity-1 spectrophotometer. IR (KBr), $\mathrm{cm}^{-1}$, 3348 (amide $\mathrm{N}-\mathrm{H}) ; 1684$ (amide, $\mathrm{C}=\mathrm{O}) ; 1499$ and $1342\left(-\mathrm{NO}_{2}\right)$

Absorption correction: CrysAlisPro, Agilent Technologies, Version 1.171.34.46 (release 25-11-2010 CrysAlis171 .NET) (compiled Nov 25 2010,17:55:46) Empirical absorption correction using spherical harmonics, implemented in SCALE3 ABSPACK scaling algorithm.

Geometry. All esds (except the esd in the dihedral angle between two 1.s. planes) are estimated using the full covariance matrix. The cell esds are taken into account individually in the estimation of esds in distances, angles and torsion angles; correlations between esds in cell parameters are only used when they are defined by crystal symmetry. An approximate (isotropic) treatment of cell esds is used for estimating esds involving l.s. planes.

Refinement. Refinement of $F^{2}$ against ALL reflections. The weighted $R$-factor $w R$ and goodness of fit $S$ are based on $F^{2}$, conventional $R$-factors $R$ are based on $F$, with $F$ set to zero for negative $F^{2}$. The threshold expression of $F^{2}>\sigma\left(F^{2}\right)$ is used only for calculating $R$-factors(gt) etc. and is not relevant to the choice of reflections for refinement. $R$-factors based on $F^{2}$ are statistically about twice as large as those based on $F$, and $R$ - factors based on ALL data will be even larger.

Fractional atomic coordinates and isotropic or equivalent isotropic displacement parameters $\left(\AA^{2}\right)$

\begin{tabular}{lllll}
\hline & $x$ & $y$ & $z$ & $U_{\text {iss }} * / U_{\text {eq }}$ \\
\hline C11 & $-0.13525(11)$ & $0.17880(10)$ & $0.1877(3)$ & $0.0345(4)$ \\
O1 & $0.3249(3)$ & $0.3758(3)$ & $0.5550(7)$ & $0.0413(11)$ \\
O2 & $0.2403(3)$ & $0.0390(3)$ & $0.4902(6)$ & $0.0417(12)$ \\
O3 & $0.3446(4)$ & $-0.0604(3)$ & $0.6933(7)$ & $0.0467(13)$ \\
$\mathrm{N} 1$ & $0.2996(4)$ & $0.2164(3)$ & $0.5084(7)$ & $0.0270(11)$ \\
$\mathrm{N} 2$ & $0.3297(4)$ & $0.0170(4)$ & $0.6117(7)$ & $0.0306(12)$ \\
C3 & $-0.0406(4)$ & $0.2702(4)$ & $0.2478(9)$ & $0.0246(12)$ \\
C2 & $0.0686(4)$ & $0.2489(4)$ & $0.3410(8)$ & $0.0266(14)$ \\
H2 & 0.0914 & 0.1852 & 0.3698 & $0.032 *$ \\
C1 & $0.1440(5)$ & $0.3226(4)$ & $0.3914(8)$ & $0.0263(13)$ \\
C6 & $0.1099(5)$ & $0.4148(4)$ & $0.3463(9)$ & $0.0344(16)$
\end{tabular}




\begin{tabular}{lllll} 
H6 & 0.1616 & 0.4650 & 0.3786 & $0.041^{*}$ \\
C5 & $-0.0006(5)$ & $0.4342(4)$ & $0.2534(10)$ & $0.0384(15)$ \\
H5 & -0.0242 & 0.4977 & 0.2241 & $0.046^{*}$ \\
C4 & $-0.0754(5)$ & $0.3611(4)$ & $0.2040(10)$ & $0.0337(14)$ \\
H4 & -0.1505 & 0.3739 & 0.1401 & $0.040^{*}$ \\
C7 & $0.2650(5)$ & $0.3091(4)$ & $0.4934(8)$ & $0.0264(13)$ \\
C8 & $0.4049(5)$ & $0.1813(4)$ & $0.6035(8)$ & $0.0250(13)$ \\
C9 & $0.4207(4)$ & $0.0857(4)$ & $0.6545(8)$ & $0.0262(13)$ \\
C10 & $0.5238(5)$ & $0.0478(4)$ & $0.7497(8)$ & $0.0313(14)$ \\
H10 & 0.5320 & -0.0174 & 0.7838 & $0.038^{*}$ \\
C11 & $0.6131(5)$ & $0.1073(5)$ & $0.7928(9)$ & $0.0346(15)$ \\
H11 & 0.6841 & 0.0831 & 0.8575 & $0.042^{*}$ \\
C12 & $0.6003(5)$ & $0.2006(4)$ & $0.7434(8)$ & $0.0339(15)$ \\
H12 & 0.6630 & 0.2406 & 0.7739 & $0.041^{*}$ \\
C13 & $0.4981(4)$ & $0.2388(4)$ & $0.6497(8)$ & $0.0298(14)$ \\
H13 & 0.4916 & 0.3041 & 0.6167 & $0.036^{*}$ \\
H1N & $0.253(6)$ & $0.161(5)$ & $0.460(10)$ & $0.07(3)^{*}$ \\
\hline
\end{tabular}

Atomic displacement parameters $\left(\AA^{2}\right)$

\begin{tabular}{lllllll}
\hline & $U^{11}$ & $U^{22}$ & $U^{33}$ & $U^{12}$ & $U^{13}$ & $U^{23}$ \\
\hline C11 & $0.0180(6)$ & $0.0357(8)$ & $0.0445(8)$ & $-0.0025(6)$ & $-0.0009(8)$ & $-0.0003(8)$ \\
O1 & $0.023(2)$ & $0.030(3)$ & $0.064(3)$ & $-0.001(2)$ & $-0.001(2)$ & $-0.002(2)$ \\
O2 & $0.021(2)$ & $0.035(3)$ & $0.061(3)$ & $-0.0019(19)$ & $-0.004(2)$ & $0.001(2)$ \\
O3 & $0.034(3)$ & $0.028(3)$ & $0.072(3)$ & $-0.002(2)$ & $0.003(2)$ & $0.012(2)$ \\
N1 & $0.017(2)$ & $0.028(3)$ & $0.032(3)$ & $-0.002(2)$ & $-0.002(2)$ & $0.000(2)$ \\
N2 & $0.017(3)$ & $0.032(3)$ & $0.041(3)$ & $-0.002(2)$ & $0.006(2)$ & $-0.001(2)$ \\
C3 & $0.018(3)$ & $0.028(3)$ & $0.028(3)$ & $-0.001(2)$ & $0.005(3)$ & $-0.002(3)$ \\
C2 & $0.018(3)$ & $0.030(3)$ & $0.031(3)$ & $0.001(3)$ & $0.004(2)$ & $0.003(2)$ \\
C1 & $0.019(3)$ & $0.031(4)$ & $0.028(3)$ & $0.000(3)$ & $0.005(2)$ & $0.003(2)$ \\
C6 & $0.023(3)$ & $0.028(4)$ & $0.048(4)$ & $-0.001(3)$ & $0.002(3)$ & $-0.001(3)$ \\
C5 & $0.024(3)$ & $0.033(4)$ & $0.053(4)$ & $0.007(2)$ & $0.000(4)$ & $0.009(4)$ \\
C4 & $0.022(3)$ & $0.039(4)$ & $0.039(3)$ & $0.007(3)$ & $0.005(3)$ & $0.005(3)$ \\
C7 & $0.022(3)$ & $0.027(3)$ & $0.027(3)$ & $-0.002(3)$ & $0.001(2)$ & $0.003(3)$ \\
C8 & $0.016(3)$ & $0.031(4)$ & $0.025(3)$ & $0.001(3)$ & $0.001(2)$ & $0.000(3)$ \\
C9 & $0.015(3)$ & $0.029(3)$ & $0.032(3)$ & $-0.004(2)$ & $0.003(2)$ & $-0.001(3)$ \\
C10 & $0.023(3)$ & $0.029(4)$ & $0.040(4)$ & $0.001(3)$ & $0.005(3)$ & $0.003(3)$ \\
C11 & $0.018(3)$ & $0.043(4)$ & $0.041(4)$ & $-0.002(3)$ & $0.003(3)$ & $-0.002(3)$ \\
C12 & $0.017(3)$ & $0.042(4)$ & $0.040(4)$ & $-0.004(3)$ & $0.005(3)$ & $-0.004(3)$ \\
C13 & $0.022(3)$ & $0.028(3)$ & $0.038(3)$ & $0.002(3)$ & $0.005(3)$ & $0.002(3)$ \\
& & & & & & \\
\hline
\end{tabular}

Geometric parameters $\left(\AA,{ }^{\circ}\right)$

\begin{tabular}{llll}
\hline $\mathrm{C} 11-\mathrm{C} 3$ & $1.735(5)$ & $\mathrm{C} 6-\mathrm{H} 6$ & 0.9500 \\
$\mathrm{O} 1-\mathrm{C} 7$ & $1.212(6)$ & $\mathrm{C} 5-\mathrm{C} 4$ & $1.381(7)$ \\
$\mathrm{O} 2-\mathrm{N} 2$ & $1.247(5)$ & $\mathrm{C} 5-\mathrm{H} 5$ & 0.9500 \\
$\mathrm{O} 3-\mathrm{N} 2$ & $1.219(6)$ & $\mathrm{C} 4-\mathrm{H} 4$ & 0.9500 \\
$\mathrm{~N} 1-\mathrm{C} 7$ & $1.376(7)$ & $\mathrm{C} 8-\mathrm{C} 9$ & $1.396(7)$
\end{tabular}




\begin{tabular}{|c|c|c|c|}
\hline $\mathrm{N} 1-\mathrm{C} 8$ & $1.405(7)$ & $\mathrm{C} 8-\mathrm{C} 13$ & $1.397(7)$ \\
\hline $\mathrm{N} 1-\mathrm{H} 1 \mathrm{~N}$ & $0.98(7)$ & $\mathrm{C} 9-\mathrm{C} 10$ & $1.397(8)$ \\
\hline $\mathrm{N} 2-\mathrm{C} 9$ & $1.474(7)$ & $\mathrm{C} 10-\mathrm{C} 11$ & $1.374(8)$ \\
\hline $\mathrm{C} 3-\mathrm{C} 4$ & $1.365(7)$ & $\mathrm{C} 10-\mathrm{H} 10$ & 0.9500 \\
\hline $\mathrm{C} 3-\mathrm{C} 2$ & $1.388(7)$ & $\mathrm{C} 11-\mathrm{C} 12$ & $1.360(8)$ \\
\hline $\mathrm{C} 2-\mathrm{C} 1$ & $1.392(7)$ & $\mathrm{C} 11-\mathrm{H} 11$ & 0.9500 \\
\hline $\mathrm{C} 2-\mathrm{H} 2$ & 0.9500 & $\mathrm{C} 12-\mathrm{C} 13$ & $1.387(7)$ \\
\hline $\mathrm{C} 1-\mathrm{C} 6$ & $1.382(7)$ & $\mathrm{C} 12-\mathrm{H} 12$ & 0.9500 \\
\hline $\mathrm{C} 1-\mathrm{C} 7$ & $1.513(8)$ & $\mathrm{C} 13-\mathrm{H} 13$ & 0.9500 \\
\hline $\mathrm{C} 6-\mathrm{C} 5$ & $1.396(7)$ & & \\
\hline $\mathrm{C} 7-\mathrm{N} 1-\mathrm{C} 8$ & $127.8(5)$ & $\mathrm{C} 5-\mathrm{C} 4-\mathrm{H} 4$ & 120.2 \\
\hline $\mathrm{C} 7-\mathrm{N} 1-\mathrm{H} 1 \mathrm{~N}$ & $126(4)$ & $\mathrm{O} 1-\mathrm{C} 7-\mathrm{N} 1$ & $124.1(5)$ \\
\hline $\mathrm{C} 8-\mathrm{N} 1-\mathrm{H} 1 \mathrm{~N}$ & $106(4)$ & $\mathrm{O} 1-\mathrm{C} 7-\mathrm{C} 1$ & $121.3(5)$ \\
\hline $\mathrm{O} 3-\mathrm{N} 2-\mathrm{O} 2$ & $121.9(5)$ & $\mathrm{N} 1-\mathrm{C} 7-\mathrm{C} 1$ & $114.6(5)$ \\
\hline $\mathrm{O} 3-\mathrm{N} 2-\mathrm{C} 9$ & $119.0(5)$ & $\mathrm{C} 9-\mathrm{C} 8-\mathrm{C} 13$ & $116.8(5)$ \\
\hline $\mathrm{O} 2-\mathrm{N} 2-\mathrm{C} 9$ & $119.1(5)$ & $\mathrm{C} 9-\mathrm{C} 8-\mathrm{N} 1$ & $120.8(5)$ \\
\hline $\mathrm{C} 4-\mathrm{C} 3-\mathrm{C} 2$ & $121.8(5)$ & $\mathrm{C} 13-\mathrm{C} 8-\mathrm{N} 1$ & $122.4(5)$ \\
\hline $\mathrm{C} 4-\mathrm{C} 3-\mathrm{Cl1}$ & $119.3(4)$ & $\mathrm{C} 8-\mathrm{C} 9-\mathrm{C} 10$ & $122.7(5)$ \\
\hline $\mathrm{C} 2-\mathrm{C} 3-\mathrm{Cl1}$ & $118.9(4)$ & $\mathrm{C} 8-\mathrm{C} 9-\mathrm{N} 2$ & $122.5(5)$ \\
\hline $\mathrm{C} 3-\mathrm{C} 2-\mathrm{C} 1$ & $118.7(5)$ & $\mathrm{C} 10-\mathrm{C} 9-\mathrm{N} 2$ & $114.8(5)$ \\
\hline $\mathrm{C} 3-\mathrm{C} 2-\mathrm{H} 2$ & 120.6 & $\mathrm{C} 11-\mathrm{C} 10-\mathrm{C} 9$ & $118.3(6)$ \\
\hline $\mathrm{C} 1-\mathrm{C} 2-\mathrm{H} 2$ & 120.6 & $\mathrm{C} 11-\mathrm{C} 10-\mathrm{H} 10$ & 120.9 \\
\hline $\mathrm{C} 6-\mathrm{C} 1-\mathrm{C} 2$ & $120.0(5)$ & $\mathrm{C} 9-\mathrm{C} 10-\mathrm{H} 10$ & 120.9 \\
\hline $\mathrm{C} 6-\mathrm{C} 1-\mathrm{C} 7$ & $116.0(5)$ & $\mathrm{C} 12-\mathrm{C} 11-\mathrm{C} 10$ & $120.3(6)$ \\
\hline $\mathrm{C} 2-\mathrm{C} 1-\mathrm{C} 7$ & $124.0(5)$ & $\mathrm{C} 12-\mathrm{C} 11-\mathrm{H} 11$ & 119.8 \\
\hline $\mathrm{C} 1-\mathrm{C} 6-\mathrm{C} 5$ & $120.0(6)$ & $\mathrm{C} 10-\mathrm{C} 11-\mathrm{H} 11$ & 119.8 \\
\hline $\mathrm{C} 1-\mathrm{C} 6-\mathrm{H} 6$ & 120.0 & $\mathrm{C} 11-\mathrm{C} 12-\mathrm{C} 13$ & $121.7(6)$ \\
\hline $\mathrm{C} 5-\mathrm{C} 6-\mathrm{H} 6$ & 120.0 & $\mathrm{C} 11-\mathrm{C} 12-\mathrm{H} 12$ & 119.2 \\
\hline $\mathrm{C} 4-\mathrm{C} 5-\mathrm{C} 6$ & $119.9(6)$ & $\mathrm{C} 13-\mathrm{C} 12-\mathrm{H} 12$ & 119.2 \\
\hline $\mathrm{C} 4-\mathrm{C} 5-\mathrm{H} 5$ & 120.0 & $\mathrm{C} 12-\mathrm{C} 13-\mathrm{C} 8$ & $120.1(5)$ \\
\hline $\mathrm{C} 6-\mathrm{C} 5-\mathrm{H} 5$ & 120.0 & $\mathrm{C} 12-\mathrm{C} 13-\mathrm{H} 13$ & 119.9 \\
\hline $\mathrm{C} 3-\mathrm{C} 4-\mathrm{C} 5$ & $119.5(5)$ & $\mathrm{C} 8-\mathrm{C} 13-\mathrm{H} 13$ & 119.9 \\
\hline $\mathrm{C} 3-\mathrm{C} 4-\mathrm{H} 4$ & 120.2 & & \\
\hline $\mathrm{O} 2-\mathrm{O} 2-\mathrm{N} 2-\mathrm{O} 3$ & $0.0(3)$ & $\mathrm{C} 7-\mathrm{N} 1-\mathrm{C} 8-\mathrm{C} 13$ & $-18.1(9)$ \\
\hline $\mathrm{O} 2-\mathrm{O} 2-\mathrm{N} 2-\mathrm{C} 9$ & $0.0(6)$ & $\mathrm{C} 13-\mathrm{C} 8-\mathrm{C} 9-\mathrm{C} 10$ & $0.9(9)$ \\
\hline $\mathrm{C} 4-\mathrm{C} 3-\mathrm{C} 2-\mathrm{C} 1$ & $0.2(9)$ & $\mathrm{N} 1-\mathrm{C} 8-\mathrm{C} 9-\mathrm{C} 10$ & $-179.8(5)$ \\
\hline $\mathrm{C} 11-\mathrm{C} 3-\mathrm{C} 2-\mathrm{C} 1$ & $-179.1(4)$ & $\mathrm{C} 13-\mathrm{C} 8-\mathrm{C} 9-\mathrm{N} 2$ & $-179.1(5)$ \\
\hline $\mathrm{C} 3-\mathrm{C} 2-\mathrm{C} 1-\mathrm{C} 6$ & $-0.8(8)$ & $\mathrm{N} 1-\mathrm{C} 8-\mathrm{C} 9-\mathrm{N} 2$ & $0.2(8)$ \\
\hline $\mathrm{C} 3-\mathrm{C} 2-\mathrm{C} 1-\mathrm{C} 7$ & $179.9(5)$ & $\mathrm{O} 3-\mathrm{N} 2-\mathrm{C} 9-\mathrm{C} 8$ & $-166.2(6)$ \\
\hline $\mathrm{C} 2-\mathrm{C} 1-\mathrm{C} 6-\mathrm{C} 5$ & $1.1(9)$ & $\mathrm{O} 2-\mathrm{N} 2-\mathrm{C} 9-\mathrm{C} 8$ & $15.1(8)$ \\
\hline $\mathrm{C} 7-\mathrm{C} 1-\mathrm{C} 6-\mathrm{C} 5$ & $-179.6(5)$ & $\mathrm{O} 2-\mathrm{N} 2-\mathrm{C} 9-\mathrm{C} 8$ & $15.1(8)$ \\
\hline $\mathrm{C} 1-\mathrm{C} 6-\mathrm{C} 5-\mathrm{C} 4$ & $-0.9(10)$ & $\mathrm{O} 3-\mathrm{N} 2-\mathrm{C} 9-\mathrm{C} 10$ & $13.8(7)$ \\
\hline $\mathrm{C} 2-\mathrm{C} 3-\mathrm{C} 4-\mathrm{C} 5$ & $0.0(10)$ & $\mathrm{O} 2-\mathrm{N} 2-\mathrm{C} 9-\mathrm{C} 10$ & $-164.8(5)$ \\
\hline $\mathrm{C} 11-\mathrm{C} 3-\mathrm{C} 4-\mathrm{C} 5$ & $179.3(5)$ & $\mathrm{O} 2-\mathrm{N} 2-\mathrm{C} 9-\mathrm{C} 10$ & $-164.8(5)$ \\
\hline $\mathrm{C} 6-\mathrm{C} 5-\mathrm{C} 4-\mathrm{C} 3$ & $0.3(10)$ & $\mathrm{C} 8-\mathrm{C} 9-\mathrm{C} 10-\mathrm{C} 11$ & $-0.6(9)$ \\
\hline $\mathrm{C} 8-\mathrm{N} 1-\mathrm{C} 7-\mathrm{O} 1$ & $3.7(10)$ & $\mathrm{N} 2-\mathrm{C} 9-\mathrm{C} 10-\mathrm{C} 11$ & $179.4(5)$ \\
\hline
\end{tabular}


supporting information

$\begin{array}{llll}\mathrm{C} 8-\mathrm{N} 1-\mathrm{C} 7-\mathrm{C} 1 & -176.5(5) & \mathrm{C} 9-\mathrm{C} 10-\mathrm{C} 11-\mathrm{C} 12 & 0.0(9) \\ \mathrm{C} 6-\mathrm{C} 1-\mathrm{C} 7-\mathrm{O} 1 & 9.1(8) & \mathrm{C} 10-\mathrm{C} 11-\mathrm{C} 12-\mathrm{C} 13 & 0.2(9) \\ \mathrm{C} 2-\mathrm{C} 1-\mathrm{C} 7-\mathrm{O} 1 & -171.5(6) & \mathrm{C} 11-\mathrm{C} 12-\mathrm{C} 13-\mathrm{C} 8 & 0.1(9) \\ \mathrm{C} 6-\mathrm{C} 1-\mathrm{C} 7-\mathrm{N} 1 & -170.6(5) & \mathrm{C} 9-\mathrm{C} 8-\mathrm{C} 13-\mathrm{C} 12 & -0.6(8) \\ \mathrm{C} 2-\mathrm{C} 1-\mathrm{C} 7-\mathrm{N} 1 & 8.7(8) & \mathrm{N} 1-\mathrm{C} 8-\mathrm{C} 13-\mathrm{C} 12 & -179.9(5) \\ \mathrm{C} 7-\mathrm{N} 1-\mathrm{C} 8-\mathrm{C} 9 & 162.7(6) & & \end{array}$

Hydrogen-bond geometry $\left(\AA,{ }^{\circ}\right)$

\begin{tabular}{lllll}
\hline$D-\mathrm{H} \cdots A$ & $D-\mathrm{H}$ & $\mathrm{H} \cdots A$ & $D \cdots A$ & $D-\mathrm{H} \cdots A$ \\
\hline $\mathrm{N} 1-\mathrm{H} 1 N \cdots \mathrm{O} 2$ & $0.98(7)$ & $1.75(7)$ & $2.612(6)$ & $144(6)$ \\
$\mathrm{C} 10-\mathrm{H} 10 \cdots \mathrm{O} 1^{\mathrm{i}}$ & 0.95 & 2.39 & $3.158(7)$ & 138 \\
\hline
\end{tabular}

Symmetry code: (i) $-x+1, y-1 / 2,-z+3 / 2$. 